\title{
Polarization memory of photoluminescence related with Si nanoparticles embedded into oxide matrix
}

\author{
K.V. Michailovska, I.Z. Indutnyi, O.O. Kudryavtsev, M.V. Sopinskyy, P.E. Shepeliavyi \\ V. Lashkaryov Institute of Semiconductor Physics, National Academy of Sciences of Ukraine \\ 41, prospect Nauky, 03028 Kyiv, Ukraine
}

\begin{abstract}
Investigated in this paper have been polarization properties of photoluminescence in solid and porous $\mathrm{nc}-\mathrm{Si}-\mathrm{SiO}_{\mathrm{x}}$ light emitting structures passivated in $\mathrm{HF}$ vapor. These structures were produced by thermal vacuum evaporation of silicon monoxide $\mathrm{SiO}$ powder onto polished $\mathrm{c}-\mathrm{Si}$ substrates. After annealing in vacuum for $15 \mathrm{~min}$ at the temperature $975^{\circ} \mathrm{C}, \mathrm{SiO}_{\mathrm{x}}$ films were decomposed to $\mathrm{SiO}_{2}$ with $\mathrm{Si}$ nanoclusters embedded in the oxide matrix. Comparison of polarizations, inherent to exciting light and that of film photoluminescence, enabled to find the polarization memory effect in the passivated structures. In anisotropic porous nc-Si-SiO ${ }_{x}$ samples, obtained by oblique deposition in vacuum, there is also well-defined orientation dependence of the PL polarization degree in the sample plane. This dependence is related to the orientation of oxide nanocolumns that form the structure of the porous layer. The above effects are associated with transformation during etching in $\mathrm{HF}$ the symmetric $\mathrm{Si}$ nanoparticles to asymmetric elongated ones.
\end{abstract}

Keywords: Si nanoparticle, oxide matrix, photoluminescence, polarized luminescence, polarization memory.

Manuscript received 26.03.15; revised version received 29.07.15; accepted for publication 03.09.15; published online 30.09.15.

\section{Introduction}

Porous silicon and thin-film nc-Si-SiO ${ }_{x}$ structures containing $\mathrm{Si}$ nanoclusters (nc-Si) embedded into the $\mathrm{SiO}_{\mathrm{x}}$ matrix attract attention of many researchers, because of their promising applications in advanced electronic and optoelectronic devices. Both materials show an intense and wide photoluminescence (PL) emission peaking in the near-infrared or visible spectrum. However, they essentially differ in polarization of their PL. In porous silicon, the polarization memory effect (PM), that is the correlation between polarization of excited light and polarization property of photoluminescence (PL), was found, and its features and mechanism were studied [1-5]. However, in thin-film nc-Si-SiO $x$ structures, in which silicon nanoparticles (nc-Si) are embedded into $\mathrm{SiO}_{x}$ matrix, the PM effect was not observed. This result was obtained for $\mathrm{nc}-\mathrm{Si}-\mathrm{SiO}_{\mathrm{x}}$ structures formed by high-temperature annealing of non-stoichiometric silicon oxide $\mathrm{SiO}_{x}$ in inert atmosphere or vacuum. Because of the isotropy of amorphous oxide, silicon nanoparticles formed during annealing are also isotropic, so the effect of PM in these structures does not manifest itself.

In the previous works, it was shown $[6,7]$ that the treatment of these structures in solution or vapors of hydrofluoric acid can significantly increase the PL intensity and shift the PL peak position to short-wave 
region due to partial etching of nc-Si and passivation of their surface. It can be assumed that this etching can change the shape of symmetric nanoparticles, similarly to that in porous silicon, and leads to the PM effect. Especially effective etching and passivation takes place in developed by us porous $\mathrm{nc}-\mathrm{Si}-\mathrm{SiO}_{\mathrm{x}}$ structures that are formed by oblique deposition of $\mathrm{Si}$ monoxide $(\mathrm{SiO})$ in vacuum and the following high-temperature annealing of obtained $\mathrm{SiO}_{\mathrm{x}}$ layer $[8,9]$. These layers have a porous columnar structure with oxide nanocolumns inclined at a certain angle to the sample surface. During hightemperature annealing of these films, the thermally stimulated formation of $\mathrm{Si}$ nanoinclusions occurs in a restricted volume of the $\mathrm{SiO}_{\mathrm{x}}$ columns. Because of free space (cavities) between the oxide columns, the structures is more susceptible to chemical treatments, e.g., to etching in HF solution or vapor $[10,11]$. These light-emitting structures demonstrate asymmetry in optical properties even before etching [12], so it seems desirable to investigate the polarization properties of their PL.

In this paper, the polarization properties of solid and porous passivated in $\mathrm{HF}$ vapour $\mathrm{nc}-\mathrm{Si}-\mathrm{SiO}_{\mathrm{x}}$ light emitting structures were investigated.

\section{Experiment}

The investigated $\mathrm{nc}-\mathrm{Si}-\mathrm{SiO}_{\mathrm{x}}$ light-emitting structures were produced by thermal evaporation of $99.9 \%$ pure silicon monoxide $\mathrm{SiO}$ (Cerac Inc.) powder in vacuum $\left((1 \ldots 2) \times 10^{-3} \mathrm{~Pa}\right)$ onto polished $\mathrm{c}-\mathrm{Si}$ substrates. The substrates were arranged at the angles $(\alpha)$ of $0^{\circ}$ and $60^{\circ}$ relatively to the normal to the substrate surface with the direction to the evaporator (normal and oblique deposition). The evaporation rate was monitored in situ by the quartz-crystal-oscillator monitor system (КИТ-1). The deposited film thickness was measured using МИИ-4 micro-interferometer and amounted 400 to $950 \mathrm{~nm}$. Because of additional oxidation by residual gases in the vacuum chamber during evaporation of $\mathrm{SiO}$, the obtained $\mathrm{SiO}_{\mathrm{x}}$ films were compositionally nonstoichiometric $(x>1)$. The films were annealed in vacuum for $15 \mathrm{~min}$ at the temperature close to $975^{\circ} \mathrm{C}$. This high-temperature annealing induces decomposition of $\mathrm{SiO}_{\mathrm{x}}$ into $\mathrm{Si}$ and $\mathrm{SiO}_{2}$ and formation of $\mathrm{Si}$ nanoclusters embedded in the oxide matrix. Passivation of the nc$\mathrm{Si}-\mathrm{SiO}_{\mathrm{x}}$ structures obtained in this manner was carried in the closed cell with HF vapor flow at the temperature $30{ }^{\circ} \mathrm{C}$ in the presence of etching-assisting ultraviolet light.

The PL spectra were excited using linearly polarized emission of a semiconductor laser (with the wavelength $415 \mathrm{~nm}$ ) at nearly normal incidence to the surface of the samples, and emitted light was collected in the direction normal to the surface. Polarization of the exciting light was rotated by a $\lambda / 2$ phase plate and cleaned by a linear polarizer. Another sheet polarizer (analyzer) was placed in the detection path. PL spectra were measured at room temperature within the wavelength range 500 to $850 \mathrm{~nm}$. These spectra were normalized to the spectral sensitivity of the experimental system and were corrected with respect to the polarization dependent response of the measurement system.

\section{Results and discussion}

The structure of obliquely deposited $\mathrm{SiO}_{\mathrm{x}}$ films was studied by SEM apparatus (ZEISS EVO 50XVP, Oberkochen, Germany) in the previous papers $[6,11]$. These films have a porous inclined pillar-like structure with the pillar (column) diameters of 10 to $100 \mathrm{~nm}$. Porosity of the films depends on the angle of deposition and equals to $34 \%$ for $\alpha=60^{\circ}$ [8], while the inclination of the formed oxide nanocolumns relatively to the normal to the sample surface was $26 \ldots 29^{\circ}$ [12]. Hightemperature annealing of these films does not change porosity and pillar-like structure of the samples. Porosity of the normally deposited $\mathrm{SiO}_{\mathrm{x}}$ films was less than $10 \%$, these films were isotropic, and after annealing they were practically nonporous [13].

Fig. 1 shows the PL spectra of $\mathrm{nc}-\mathrm{Si}-\mathrm{SiO}_{\mathrm{x}}$ sample deposited in vacuum at the angle $0^{\circ}$, annealed at $975^{\circ} \mathrm{C}$ in vacuum, and then etched in HF vapor (curves 1 and 2). The curve 1 corresponds to the orientation of the analyzer, which selects polarization of PL parallel to polarization of exciting radiation, and the curve 2 orientation of the analyzer that is perpendicular to polarization of excitation. As seen from the figure, the intensity of PL polarized in parallel to polarization of excitation (curve 1) is much higher than the PL component polarized in the perpendicular direction (curve 2), i.e., in the investigated sample the PM effect is really observed.

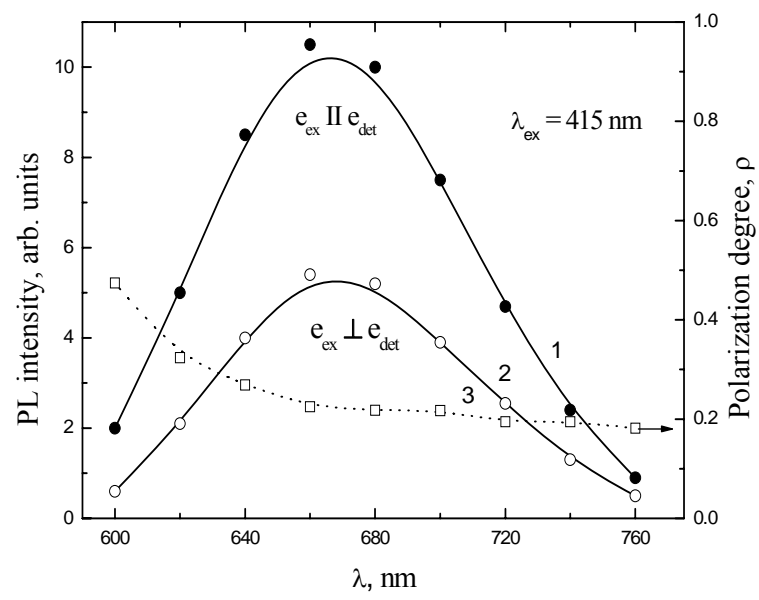

Fig. 1. PL spectra of solid $n c-S i-\mathrm{SiO}_{x}$ sample annealed in vacuum, then treated with $\mathrm{HF}$ vapors $(1,2)$. The curve 1 corresponds to parallel orientation of the analyzer to polarization of the exciting radiation, and the curve 2 orientation of the analyzer in the perpendicular direction. The curve 3 - value of degree of the linear polarization of PL for this sample. 
The effect of PM can be illustrated by the degree of linear polarization of the PL, which is known to be defined with the expression:

$\rho=\frac{I_{\perp}-I_{\perp}}{I_{\mid}+I_{\perp}}$.

Here, $I_{\|}$is the intensity of the photoluminescence polarized in parallel to that of the excited light and $I_{\perp}-$ intensity of photoluminescence polarized in the perpendicular direction. The curve 3 in Fig. 1 corresponds to the $\rho$ value inherent to the investigated sample. Near to the PL maximum, $\rho$ has the value close to 0.2 and increases in the short-wave region of the spectrum, which is similar to the results for porous silicon $[2,3]$.

Fig. 2 shows the PL intensity dependence on the angle between polarization of the exciting radiation and orientation of the analyzer for the same sample as in Fig. 1, recorded near the PL peak $(700 \mathrm{~nm})$. The curves 1 and 2 correspond to two mutually perpendicular orientations of the excitation polarization. For these orientations, the $\rho$ values are equal to 0.23 and 0.22 , i.e., they coincide within the measurement errors, which indicates that the PM effect is isotropic in the plane of the sample.

As shown by previous investigations in similar solid $\mathrm{nc}-\mathrm{Si}-\mathrm{SiO}_{\mathrm{x}}$ samples annealed in vacuum or inert gas, but not processed in HF, there is much weaker PL and no PM effect. Thus, within the measurement errors $\rho=0$ throughout the investigated range of PL spectra. Similar results were obtained for the sample of porous nc$\mathrm{Si}-\mathrm{SiO}_{\mathrm{x}}$, produced using vacuum deposition of $\mathrm{SiO}$ at the angle $60^{\circ}$ and annealed for $15 \mathrm{~min}$. The spectral dependence of PL obtained at orientation of the analyzer parallel and perpendicular to the polarization of the exciting radiation coincide, and the $\rho$ value is zero over all the studied spectral range. These results were obtained for any orientation of the excitation polarization relatively to the projection of the inclined $\mathrm{SiO}_{\mathrm{x}}$ nanocolumns. Thus, in oblique deposited porous $\mathrm{nc}-\mathrm{Si}-\mathrm{SiO}_{\mathrm{x}}$ structures that exhibit optical anisotropy due to tilting the $\mathrm{SiO}_{\mathrm{x}}$ columns [12], the PM effect is not manifested, too.

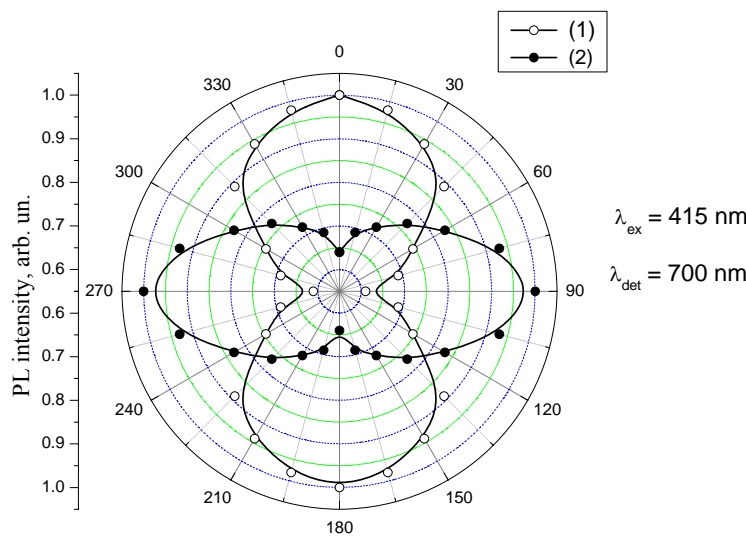

Fig. 2. Polar plot of the PL intensity for $n c-S i-\mathrm{SiO}_{x}$ sample, which spectra are shown in Fig. 1 for two mutually perpendicular orientations of excitation polarization $(1,2)$. The $\mathrm{PL}$ wavelength is $700 \mathrm{~nm}$.

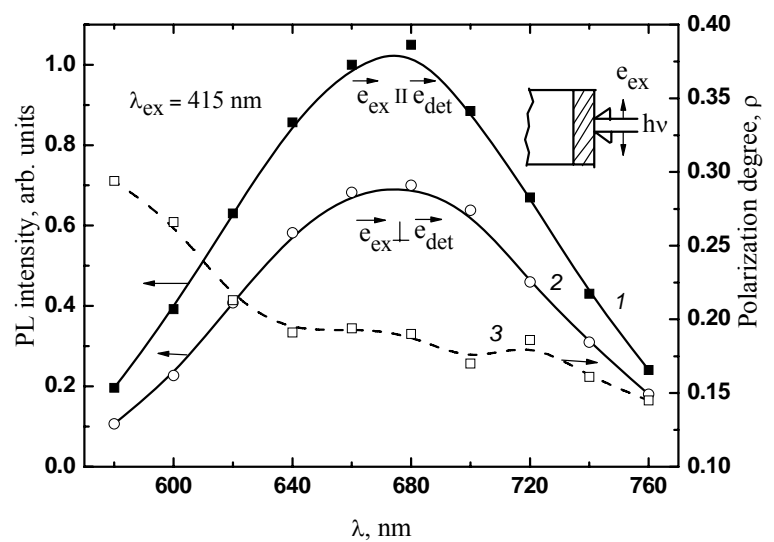

Fig. 3. $\mathrm{PL}$ spectra of porous $\mathrm{nc}-\mathrm{Si}-\mathrm{SiO}_{\mathrm{x}}$ sample annealed in vacuum, then treated with HF vapors for parallel (1) and perpendicular (2) orientations of the analyzer relatively to polarization of exciting radiation, which is oriented in parallel to the projection of $\mathrm{SiO}_{\mathrm{x}}$ nanocolumns on the sample plane. $3-$ value of the degree of PL linear polarization.

But after treatment in HF vapor, the luminescent properties of $\mathrm{nc}-\mathrm{Si}-\mathrm{SiO}_{\mathrm{x}}$ structures are changed, in particular, there is a significant shift of the PL maximum to the visible part of spectrum and increase in the PL intensity. These changes are explained by decrease in the nc-Si size during etching and passivation of the surface [9]. Besides that, the dependence of PL polarization on excitation polarization direction appears in the porous nc-Si-SiO ${ }_{x}$ structures, just as in solid samples. Fig. 3 shows PL spectra of the porous $\mathrm{nc}-\mathrm{Si}-\mathrm{SiO}_{\mathrm{x}}$ sample obtained by deposition at the angle $60^{\circ}$ and annealed at $975{ }^{\circ} \mathrm{C}$ in vacuum for $15 \mathrm{~min}$, then treated with $\mathrm{HF}$ vapor. The curves 1 and 2 correspond to orientation of the analyzer parallel and perpendicular to polarization of the exciting radiation, which is oriented in parallel to the projection of the inclined $\mathrm{SiO}_{\mathrm{x}}$ nanocolumns on the sample plane. It can be seen that the intensity of PL component polarized in parallel to polarization of the exciting radiation is substantially higher than the intensity of PL polarized in the perpendicular direction. The curve 3 in this figure represents the degree of PL linear polarization.

But unlike results shown in Fig. 1, the PM effect on the porous $\mathrm{nc}-\mathrm{Si}-\mathrm{SiO}_{\mathrm{x}}$ structures has its peculiarities. Fig. 4 shows the PL spectra of the same sample as in Fig. 3, but for the case of excitation polarization perpendicular to the projection of inclined $\mathrm{SiO}_{\mathrm{x}}$ nanocolumns on the sample plane. It can be seen that the difference between $I_{\|}$and $I_{\perp}$ is significantly smaller as compared with the case where this sample during measurement was fixed at the position when polarization of the exciting light coincides with the direction of nanocolumns projection.

More clearly, PM anisotropy in the sample plane is manifested in dependences of the PL intensity on the angle between polarization of the exciting radiation and orientation of the analyzer. Fig. 5 shows such 
dependences for two orientations of the excitation polarization relatively to the projection of $\mathrm{SiO}_{x}$ nanocolumns. For parallel orientation of the excitation polarization and nanocolumns projection, the PM effect is pronounced much efficiently than for perpendicular orientation, and $\rho$ values at the PL maximum are equal to 0.19 and 0.09 , respectively.

This result is similar to that observed in porous silicon formed by electrochemical etching of $\mathrm{Si}$ with orientation [100] in the presence of linearly polarized light illumination [2], which indicated the existence of PM anisotropy in the plane of the sample.

It was proposed several mechanisms that determine the PM effect. In [14], PL polarization anisotropy of elongated particles associated with the structure of the valence band due to quantum confinement in two directions - parallel and perpendicular to the longer axis of the particle. More common explanation - within the dielectric model in which porous silicon is considered as a

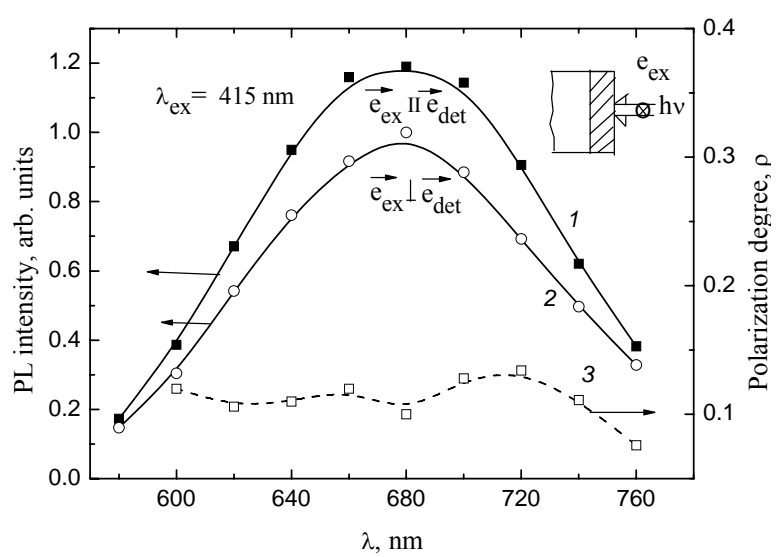

Fig. 4. PL spectra of the same sample as in Fig. 3, but for orientation of excitation polarization perpendicular to the projection of $\mathrm{SiO}_{\mathrm{x}}$ nanocolumns on the sample plane.

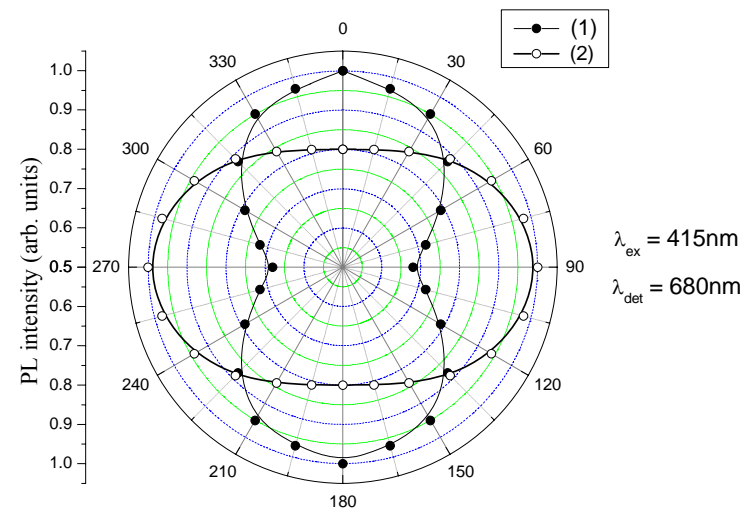

Fig. 5. Polar plot of the PL intensity for $\mathrm{nc}-\mathrm{Si}-\mathrm{SiO}_{\mathrm{x}}$ sample, which spectra are shown in Figs 3 and 4 for two mutually perpendicular orientations of excitation polarization parallel orientation of the excitation polarization and nanocolumns projection (1) and perpendicular orientation (2). The PL wavelength is $680 \mathrm{~nm}$.

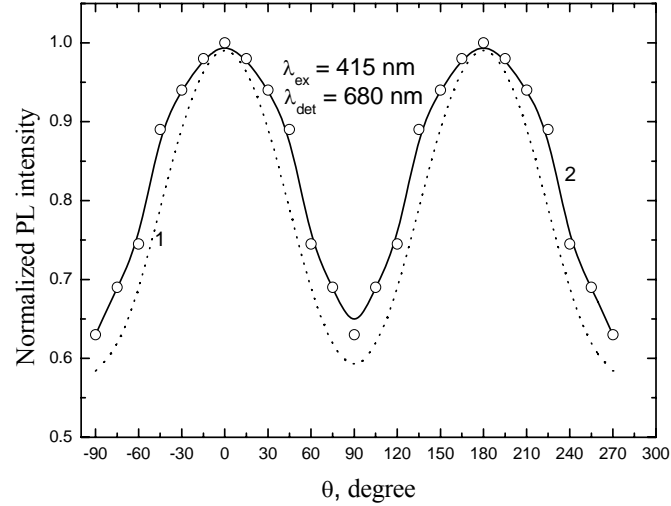

Fig. 6. Dependence of the normalized PL intensity on the angle $(\theta)$ between excitation and detection polarizations for continuous $\mathrm{nc}-\mathrm{Si}-\mathrm{SiO}_{\mathrm{x}}$ sample annealed in vacuum, then treated with $\mathrm{HF}$ vapor. 1 - simulation results, 2 experimental values. The PL wavelength $-680 \mathrm{~nm}$.

composite that includes elongated and flattened silicon nanocrystals preferentially oriented as elongated nc-Si along the [100] direction [1-3]. The probability of optical absorptions and emission is proportional to the square of the electric field inside the nc-Si and, therefore, nanocrystals with their longest dimensions aligned along the polarization direction of exciting light will preferentially absorb and emit photons. Then PM is the result of selective excitation that part of the nonspherical silicon nanoparticles whose longer axis is parallel to polarization of exciting radiation [1, 4, 15]. Both interpretations as on the basis of quantum size effects and within the dielectric model associate the PM effect with asymmetric, elongated nanoparticles that emit PL.

Another necessary condition to observe this effect is a significant dielectric contrast between the nanoparticles and their environment. As shown experimentally in the work [16] by reducing the dielectric contrast between InP and $\mathrm{ZnO}$ nanofiber using $\mathrm{Ta}_{2} \mathrm{O}_{5}$ deposition, the authors managed to reduce the value of $\rho$ by $84 \ldots 86 \%$. In the same paper, the model was developed for calculation of the PL intensity dependence on the angle between the directions of exciting light polarization and that of detected one for the structure that contains nanowires (or elongated nanoparticles) randomly oriented in the plane of the sample (similar to our continuous $\mathrm{nc}-\mathrm{Si}-\mathrm{SiO}_{\mathrm{x}}$ structures processed in HF). The main parameters of this model is the dielectric constants of the emitting nanoparticles (or nanofibers) and dielectric environment in which these emitters are embedded. Fig. 6 shows the angular dependence of the normalized PL intensity (points experimental values, solid line - approximation of the experiment using polynomial) for continuous $\mathrm{nc}-\mathrm{Si}-\mathrm{SiO}_{\mathrm{x}}$ sample annealed in vacuum, then processed in HF vapor, the same as presented in Figs 1 and 2. The dotted curve shows the results of simulation that obtained using the expression [16]: 


$$
\langle I\rangle=\frac{\pi E^{2} L^{2}\left[\left(10 \varepsilon_{e}^{2}+4 \varepsilon_{e} \varepsilon+2 \varepsilon^{2}\right)\left(9 \varepsilon_{e}^{2}+2 \varepsilon_{e} \varepsilon+\varepsilon^{2}\right)+\left(\varepsilon_{e}-\varepsilon\right)\left(2 \varepsilon_{e}+\varepsilon\right)^{2} \cos 2 \theta\right]}{4\left(\varepsilon_{e}+\varepsilon\right)^{2}\left(3 \varepsilon_{e}^{2}+2 \varepsilon_{e} \varepsilon+\varepsilon^{2}\right)}
$$

where $\varepsilon_{e}$ and $\varepsilon$ are dielectric functions of environment and emitting nanoparticles, accordingly, $\theta$ is the angle between excitation and detection.

The value of the dielectric function $\varepsilon$ for silicon nanoparticles was calculated taking into account their size [17] and was equal to 8.8. For $\mathrm{SiO}_{\mathrm{x}}$ matrix, if taking into account its porosity and composition, $\varepsilon_{e}=1.96$ [13]. One can see minor differences between the experimental and calculated curves, which indicates adequacy of the model and close values of selected parameters to the real values of the dielectric functions for the nanoparticles and matrix.

\section{Conclusion}

In the samples deposited at the angle $0^{\circ}$, thermostimulated decomposition of $\mathrm{SiO}_{\mathrm{x}}$ and formation of nc-Si occur in the isotropic continuous film, therefore nanoparticles also have isotropic (nearly spherical) shape, and the PM effect is not observed. In porous samples deposited at the angle $60^{\circ}$, which are optically anisotropic due to inclination of $\mathrm{SiO}_{\mathrm{x}}$ nanocolumns, the PM effect is not observed, too. It means that silicon nanoparticles formed in the $\mathrm{SiO}_{\mathrm{x}}$ columns during annealing are also spherically symmetrical, and polarization of PL is mainly determined by the shape of particles that emit light. Anisotropy of the dielectric matrix is not manifested through the dependence of PL polarization on the excitation polarization.

After treatment with HF, the luminescent properties of $\mathrm{nc}-\mathrm{Si}-\mathrm{SiO}_{\mathrm{x}}$ structures are changed. The results obtained in this paper show that this etching leads not only to the reduced sizes of silicon nanoparticles, but also to changes in their shape to the elongated anisotropic one. In the continuous nc-Si- $\mathrm{SiO}_{\mathrm{x}}$ structures, in the plane of the sample there are no preferrential orientations of anisotropic silicon nanoparticles. But in the porous matrix, anisotropy of PM is observed in the plane of substrate. It means that anisotropic elongated silicon nanoparticles have preferred orientation in the film, and the projection of this orientation on the plane of the sample coincides with the direction of projection of the $\mathrm{SiO}_{\mathrm{x}}$ nanocolumns. It has been assumed that orientation of the longer axes in nc-Si coincides with orientation of $\mathrm{SiO}_{\mathrm{x}}$ nanocolumns. This assumption is consistent with the conditions of etching in porous matrix: dissolution occurs starting from the surface of the nanocolumns, so first the side surface of nc-Si is dissolved, since it is closer to the column surfaces, which leads to elongation of nanoparticles along the axis of the column.

\section{References}

1. D. Kovalev, M. Ben Chorin, J. Diener et al., Porous $\mathrm{Si}$ anisotropy from photoluminescence polarization, // Appl. Phys. Lett. 67, p. 1585-1587 (1995).

2. G. Polisski, A.V. Andrianov, D. Kovalev and F. Koch, Light-stimulated anisotropy in porous silicon // Brazil. J. Phys. 26, p. 189-192 (1996).

3. J. Diener, D. Kovalev, G. Polisski, N. Künzner and F. Koch, Morphology of porous silicon layers deduced from polarization memory experiments // phys. status solidi (b), 224, p. 297-300 (2001).

4. B. Bruhn, J. Valenta and J. Linnros, Controlled fabrication of individual silicon quantum rods yielding high intensity, polarized light emission // Nanotechnology, 20, 505301 (2009).

5. J. Valenta, R. Juhasz, J. Linnros, Photoluminescence from single silicon quantum dots at room temperature // J. Lumin. 98, p. 15-22 (2002).

6. I.Z. Indutnyi, E.V. Michailovska, P.E. Shepeliavyi, V.A. Dan'ko, Visible photoluminescence of selective etched porous $\mathrm{nc}-\mathrm{Si}-\mathrm{SiO}_{\mathrm{x}}$ structures // Fizika i Tekhnika Poluprovodnikov, 44, p. 218-221 (2010), in Russian.

7. K. Sato and K. Hirakuri, Improved luminescence intensity and stability of nanocrystalline silicon due to the passivation of nonluminescent states // J. Appl. Phys. 97(10), 104326 (2005).

8. V.A. Dan'ko, I.Z. Indutnyy, I.Y. Maidanchuk, V.I. Min'ko, P.E. Shepeliavyi, V.O. Yukhimchuk, Formation of the photoluminescence structure based on $\mathrm{SiO}_{\mathrm{x}}$ porous films // Optoelectronika $\mathrm{i}$ poluprovodnikovaya tekhnika, 39, p. 65-72 (2004) (in Ukrainian).

9. V.A. Dan'ko, V.Ya. Bratus', I.Z. Indutnyi, I.P. Lisovskyy, S.O. Zlobin, K.V. Michailovska, P.E. Shepeliavyi, Controlling the photoluminescence spectra of porous $\mathrm{nc}-\mathrm{Si}-\mathrm{SiO}_{\mathrm{x}}$ structures by vapor treatment // Semiconductor Physics, Quantum Electronics \& Optoelectronics, 13, p. 413-417 (2010).

10. J. Heitmann, F. Müller, M. Zacharias, U. Gösele, Silicon nanocrystals: Size matters // Adv. Mater. 17, p. 795-803 (2005).

11. I.Z. Indutnyi, K.V. Michailovska, V.I. Min'ko, P.E. Shepeliavyi, Effect acetone vapor treatment on photoluminescence of porous nc-Si- $\mathrm{SiO}_{\mathrm{x}}$ nanostructures // Semiconductor Physics, Quantum Electronics \& Optoelectronics, 12, p. 105-109 (2009). 
12. M.V. Sopinskyy, I.Z. Indutnyi, K.V. Michailovska, P.E. Shepeliavyi, V.M. Tkach, Polarization conversion effect in obliquely deposited $\mathrm{SiO}_{\mathrm{x}}$ films // Semiconductor Physics, Quantum Electronics \& Optoelectronics, 14, p. 273-278 (2011).

13. I.P. Lisovskyy, I.Z. Indutnyy, B.N. Gnennyy et al., Structural and phase changes in $\mathrm{SiO}_{\mathrm{x}}$ films under thermal vacuum treatments // Fizika i Tekhnika Poluprovodnikov 37, p. 98-103 (2003), in Russian.

14. W.H. Zheng, Jian-Bai Xiay and K W Cheahz, Linear polarization of photoluminescence in quantum wires // J. Phys.: Condens. Matter, 9, p. 5105-5116 (1997).
15. H.E. Ruda and A. Shik, Polarization-sensitive optical phenomena in semiconducting and metallic nanowires // Phys. Rev. B, 72, 1153081-11 (2005).

16. Lei Fang, Xianwei Zhao, Yi-Hsin Chiu, Dongkyun Ko et al., Comprehensive control of optical polarization anisotropy in semiconducting nanowires // Appl. Phys. Lett. 99, p. 141101-1141101-3 (2011).

17. C.Y. Ng, T.P. Chen, L. Ding, Y. Liu et al., Static dielectric constant of isolated silicon nanocrystals in $\mathrm{a} \mathrm{SiO}_{2}$ thin film // Appl. Phys. Lett. 88, 063103 (2006). 